\title{
Effect of differentiation on microRNA expression in bovine skeletal muscle satellite cells by deep sequencing
}

Wei Wei Zhang ${ }^{1,2}$, Xiao Feng Sun ${ }^{1}$, Hui Li Tong ${ }^{1}$, Ya Hui Wang ${ }^{1}$, Shu Feng Li ${ }^{1}$, Yun Qin Yan ${ }^{1 *}$ and Guang Peng Li ${ }^{3}$

\author{
* Correspondence: \\ yanyunqin@sohu.com \\ ${ }^{1}$ The Laboratory of Cell and \\ Development, Northeast \\ Agricultural University, Mucai Street \\ 59, Xiangfang District, Harbin \\ 150030, Heilongjiang, China \\ Full list of author information is \\ available at the end of the article
}

\begin{abstract}
Background: The differentiation of skeletal muscle-derived satellite cells (MDSCs) is important in controlling muscle growth, improving livestock muscle quality, and healing of muscle-related disease. MicroRNAs (miRNAs) are a class of gene expression regulatory factors, which play critical roles in the regulation of muscle cell differentiation. This study aimed to compare the expression profile of miRNAs in MDSC differentiation, and to investigate the miRNAs which are involved in MDSC differentiation.

Method: Total RNA was extracted from MDSCs at three different stages of differentiation (MDSC-P, MDSC-D1 and MDSC-D3, representing 0, 1 and 3 days after differentiation, respectively), and used to construct small RNA libraries for RNA sequencing (RNA-seq).
\end{abstract}

Results: The results showed that in total 617 miRNAs, including 53 novel miRNA candidates, were identified. There were 9 up-expressed, 165 down-expressed, and 15 up-expressed, 145 down-expressed in MDSC-D1 and MDSC-D3, respectively, compared to those in MDSC-P. Also, 17 up-expressed, 55 down-expressed miRNAs were observed in MDSC-D3 compared to those in MDSC-D1. All known miRNAs belong to 237 miRNA gene families. Furthermore, we observed some sequence variants and base edits of the miRNAs. GO and KEGG pathway analysis showed that the majority of target genes regulated by miRNAs were involved in cellular metabolism, pathways in cancer, actin cytoskeleton regulation and the MAPK signaling pathway. Regarding the 53 novel miRNAs, there were 7 up-expressed, 31 down-expressed, and 8 up-expressed, 26 down-expressed in MDSC-D1 and MDSC-D3, respectively, compared to those in MDSC-P. The expression levels of 12 selected miRNA genes detected by RT-qPCR were consistent with those generated by deep sequencing.

Conclusions: This study confirmed the authenticity of 564 known miRNAs and identified 53 novel miRNAs which were involved in MDSC differentiation. The identification of novel miRNAs has significantly expanded the repertoire of bovine miRNAs and could contribute to advances in understanding muscle development in cattle.

Keywords: Bovine, Skeletal muscle-derived satellite cells, Deep sequencing, miRNA, Differentiation, Proliferation, Differentially expressed miRNA, Target prediction, Gene Ontology, KEGG analysis

\section{Biomed Central}

(c) 2016 The Author(s). Open Access This article is distributed under the terms of the Creative Commons Attribution 4.0 International License (http://creativecommons.org/licenses/by/4.0/), which permits unrestricted use, distribution, and reproduction in any medium, provided you give appropriate credit to the original author(s) and the source, provide a link to the Creative Commons license, and indicate if changes were made. The Creative Commons Public Domain Dedication waiver (http://creativecommons.org/ publicdomain/zero/1.0/) applies to the data made available in this article, unless otherwise stated. 


\section{Background}

In livestock, all muscle fibers are formed during the prenatal stage. Postnatal growth and regenerative ability of adult skeletal muscles are dependent on adult muscle stem cells known as satellite cells, which reside beneath the basal lamina of the mature fibers $[1,2]$. In the process of MDSC differentiation, progenitor cells first proliferate, then exit from the cell cycle, and undergo differentiation, alignment, and fusion to form multinucleated myotubes [3-5]. These molecular events are orchestrated by myogenic regulatory factors and miRNAs. The miRNAs are a family of noncoding small RNAs, approximately 22 nucleotides in length. They can repress the translation and accelerate the decay of mRNAs through pairing of their seed sequences with the 3' UTRs of target genes [6]. The miRNAs have been shown to play critical roles in skeletal muscle development and in regulation of muscle cell proliferation and differentiation [7]. For example, miR-1 and miR-206 could promote the differentiation of myoblasts, whereas miR-133 could promote cell proliferation [8-10]. Additionally, miR-27 could modulate the entry of cells into the myogenic differentiation program [11].

Recently, Sun et al. identified conserved and novel miRNAs from the Chinese Qinchuan bovine longissimus thoracis by high-throughput sequencing [12]. There have been few studies on their involvement in the regulation of MDSC differentiation. The aim of this study was to investigate the miRNA expression profiles in MDSCs during the differentiation process by using RNA-seq. Elucidation of the expression patterns of different miRNA among different differentiation stages would contribute to our understanding of the roles of miRNAs in gene expression regulatory networks during the differentiation of MDSCs.

\section{Methods}

\section{Ethics statement}

Sample collection from animals was approved by the Animal Care Commission of the Northeast Agricultural University and Heilongjiang, P.R. China. Skeletal muscle tissues of the newborn Chinese Simmental calves were collected from Shuangcheng abattoir, a local slaughterhouse in Heilongjiang, Peoples Republic of China.

\section{MDSC culture and differentiation}

MDSCs were isolated from hindlimb muscles of three newborn Chinese Simmental calves according to the method described by Lee et al. [13] and Tong et al. [14]. The MDSCs were cultured in growth medium (GM). For MDSC differentiation, cells were seeded on plates at a density of $4-10^{5}$ cells/60-mm dishes, and allowed to adhere for $24 \mathrm{~h}$ in GM. Subsequently, the cells were switched to differentiation medium (DM) containing $2 \%$ horse serum (Gibco), $100 \mathrm{U} / \mathrm{mL}$ penicillin, and $100 \mu \mathrm{g} / \mathrm{mL}$ streptomycin in Dulbecco's modified Eagle's medium. The MDSCs were collected after switching to DM for 0 days (MDSC-P), 1 day (MDSC-D1) and 3 days (MDSC-D3). Each differentiation stage had three replicates which came from the three cattle mentioned above. The differentiation of MDSCs was evaluated by the immunolocalization of MHC and desmin genes according to the method described by Tong et al. [14], and the relative mRNA expression levels of PAX3, MYOD, MYF5, MYF6, MYOG and MHC genes which were involved in the cell differentiation. All reactions were performed in 
triplicate and the relative expression level of mRNA was normalized to the expression of the $\beta$-actin gene. The quantification of each mRNA relative to the $\beta$-actin gene was calculated by the formula: $\mathrm{N}=2^{-\Delta \Delta \mathrm{Ct}}$.

\section{RNA extraction and high-throughput sequencing}

Total RNA was extracted from MDSC samples using TRI reagent (Invitrogen, Carlsbad, CA) following the manufacturer's instructions. The quantity of total RNA was measured using an Agilent 2100 Bioanalyzer with Agilent RNA 6000 nano Reagents Port 1 kit (Agilent Technologies, Santa Clara, CA, USA). Optical density values at 260/280 were consistently above 1.9. The samples with intact, distinct ribosomal peaks were chosen for further analysis. The RNAs from three replicates were pooled as one RNA sample at each differentiation stage of MDSCs. Subsequently, the RNAs with low molecular weight were separated by $15 \%$ PAGE, and the RNAs with molecules in the range of 18-30 nt were enriched and ligated with proprietary adapters to the $5^{\prime}$ and 3 ' termini. A reverse transcription reaction followed by low-cycle PCR was performed to obtain sufficient product for high-throughput sequencing in Beijing Genomics Institute, China.

\section{Small RNA sequence analysis}

After removing the 3' adaptor sequence and removal of redundancy and reads smaller than $18 \mathrm{nt}$, the clean reads were screened against and mapped to the latest bovine genome assembly (ftp://hgdownload.cse.ucsc.edu/goldenPath/bosTau7/bigZips/bosTau7.fa.gz) using the program SOAP [15]. To identify sequences originating from protein-coding genes, repeats, rRNA, tRNA, snRNA, and snoRNA, the bovine mRNA (ftp://hgdownload.cse.ucsc.edu/ goldenPath/bosTau7/database/refGene.txt.gz) and CDS, Repeat Masker (ftp://hgdownload. cse.ucsc.edu/goldenPath/bosTau6/bigZips/bosTau7.fa.out.gz) and Sanger Rfam data (version 10.1) were used. The remaining reads were searched against the Sanger miRBase (version 21.0) database to identify conserved miRNAs. Only those small RNAs whose mature and precursor sequences perfectly matched known bovine miRNAs in miRBase were considered to be conserved miRNAs. To discover potential novel miRNA precursor sequences, unique sequences that had more than 10 hits to the genome or matched known noncoding RNAs were removed. Subsequently, the flanking sequences (150 nt upstream and downstream) of each unique sequence were extracted for secondary structure analysis with Mfold (http://www.bioinfo.rpi.edu/applications/mfold) and then evaluated by Mireap (http:// sourceforge.net/projects/mireap/). The criteria [16] were used to screen candidates for potential miRNAs or pre-miRNAs as follows: [1] pre-miRNA sequences could fold into an appropriate hairpin secondary structure that contained the $\sim 22$ nt mature miRNA sequence within one arm of the hairpin. [2] miRNA precursors with secondary structures had higher negative minimal free energies (MFEs) and minimal free energy indexes (MFEIs) than other different types of RNAs. [3] miRNA had an AU content of 30-70\%. [4] miRNA had less than six mismatches with the opposite miRNA* sequence in the other arm. [5] No loop or break in miRNA sequences was allowed. After prediction, the resulting potential miRNA loci were examined carefully based on the distribution and numbers of small RNAs in the entire precursor regions. Those sequences residing in the stem region of the stem-loop structure and ranging between 20 and 22 nt with free energy hybridization lower than $-20 \mathrm{kcal} / \mathrm{mol}$ were considered [17]. 
To predict the target genes of miRNAs, we used the RNAhybrid software program for target prediction (ftp://hgdownload.cse.ucsc.edu/goldenPath/bosTau7/bigZips/refMrna.fa.gz). This program was based on the criteria suggested by Allen et al. [18] and Schwab et al. [19]. Finally, to reveal the functions of the putative target genes, Gene Ontology (GO) analysis was performed on the predicted target gene candidates of the novel miRNAs using three ontologies: molecular function, cellular components, and biological process. The functions of the putative target genes which were regulated by miRNAs were determined using KEGG pathway analysis.

\section{MicroRNA expression analysis}

Expression levels of known miRNAs between two samples were compared to identify differentially expressed miRNAs. The expression of miRNA was shown in two samples by plotting the $\log _{2}$ ratio and making a scatter plot. The procedures were as follows. [1] Normalize the expression of miRNA in three samples (MDSC-P, MDSC-D1, and MDSC-D3) to obtain the expression of transcripts per million. Normalized expression $(\mathrm{NE})=$ actual miRNA count/total count of clean reads. [2] Calculate the fold-change and P-value from the normalized expression. Then, generate the $\log _{2}$ ratio plot and scatter plot. Fold-change formula: Fold-change formula: fold-change $=\log _{2}$ (MDSC-D1 or MDSC-D3/MDSC-P). P-value formula: $p(x / b)=\left(\frac{N_{2}}{N_{1}}\right)^{y} \frac{(x+y) !}{x ! y !\left(1+\frac{N_{2}}{N_{1}}\right)^{(x+y+1)}} \quad C\left(y \leq y_{\min } / x\right)=\sum_{y=0}^{y \leq y_{\min }} p(y / x)$, where $x$ and $y$ represent normalized expression levels, and $N_{1}$ and $N_{2}$ represent total counts of clean reads of a given miRNA in small RNA libraries of the MDSC-P, MDSC-D1, and MDSC-D3 stages, respectively.

\section{RT-qPCR}

Twelve miRNAs were randomly chosen for verification of RNA-seq results by RT-qPCR [20]. The same RNA samples used for RNA-seq library preparation and sequencing were used for the RT-qPCR. Total RNA $(1 \mu \mathrm{g})$ was converted to cDNA with an RT primer mixture (250 nM) using the BioTake Super RT Kit (BioTake, Beijing, China). The cDNA was then used for qPCR of miRNA using the miRNA-specific primer and the universal primer. The bovine $18 \mathrm{~S}$ ribosomal RNA (18S) gene was used as a reference gene. The primers for miRNAs and the reference gene are listed in Additional file 1. RT-qPCR reaction was performed on an ABI7300 Real Time Detection System. The cycle conditions were as follows: $95{ }^{\circ} \mathrm{C}$ for $2 \mathrm{~min}$, followed by 40 cycles of $95^{\circ} \mathrm{C}$ for $15 \mathrm{~s}$, and $60{ }^{\circ} \mathrm{C}$ for $30 \mathrm{~s}$. The miRNA expression was normalized to the expression of the 18S rRNA gene and calculated using the $2^{-\Delta \Delta \mathrm{Ct}}$ method. All reactions were performed in triplicate, and the RT-qPCR results among different treatment groups were statistically tested using one-way ANOVA by Tukey's test at $p=0.05$.

\section{Results}

\section{Evaluation of MDSC differentiation}

Cell differentiation was evaluated by morphology, MHC and desmin immunolocalization, as well as MRF gene expression. Bovine MDSCs were derived from the primary 
culture of muscle tissue and fused myotubes were generated by culturing MDSCs in DM for 1 and 3 days (Fig. 1a). In MDSC-P, the MHC and desmin genes were not expressed, but they were in MDSC-D1 and MDSC-D3 (Fig. 1b, c). The analysis of MRF gene expression showed the PAX3 and MYF5 genes were down-regulated, while MYOG, MYF6, and MHC genes were up-regulated in MDSC-D1 and MDSC-D3 compared to those in MDSC-P (Fig. 1d). These results indicated that the MDSCs were in

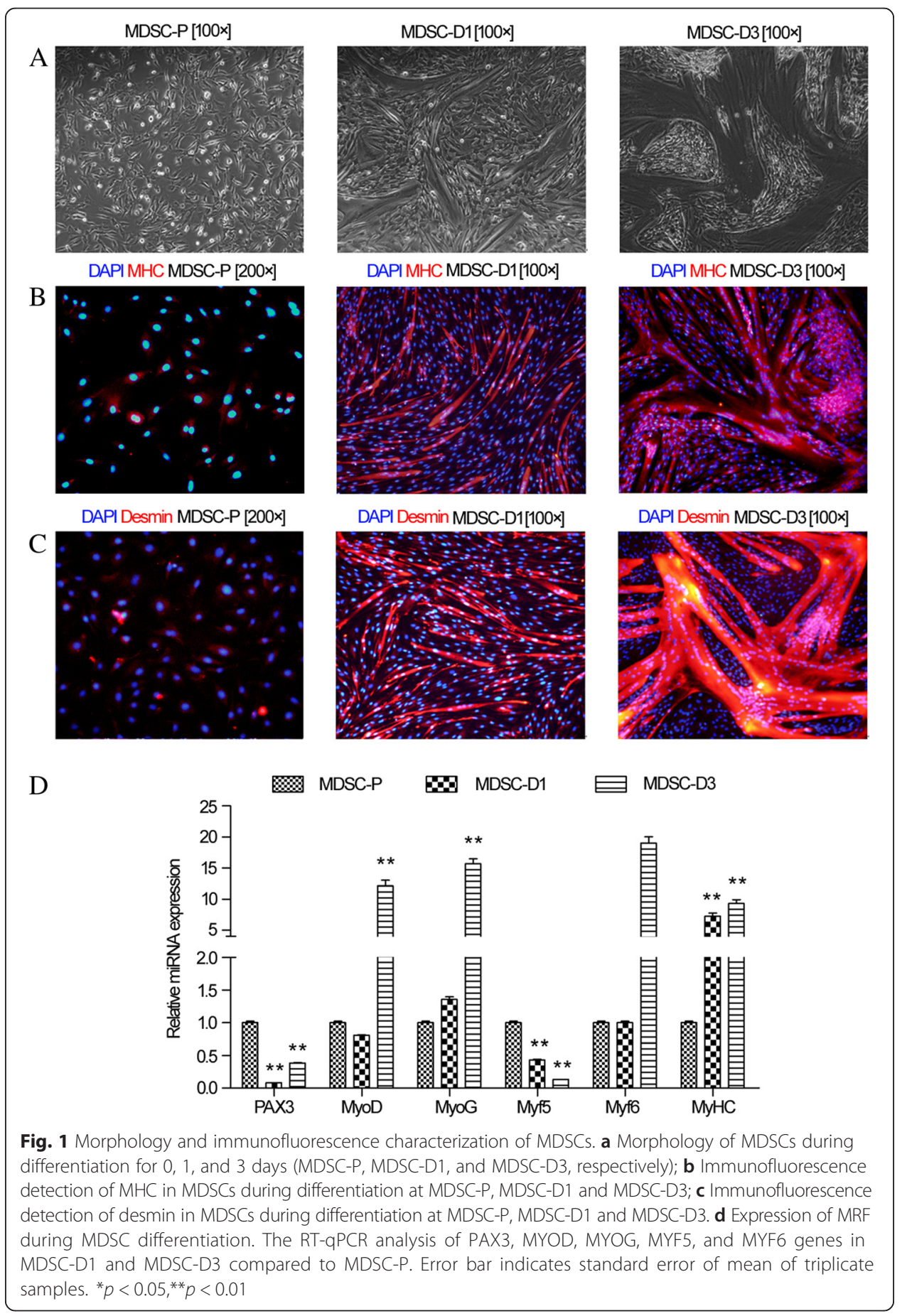


the differentiation process in MDSC-D1 and MDSC-D3 but not MDSC-P, in which the MDSCs were only in proliferation.

\section{Cell collection and high-throughput sequencing of small RNAs}

To identify small RNAs in MDSCs during differentiation, total RNAs from MDSCs at different differentiation stages were used to construct small RNA libraries. We obtained 5,871,055 clean reads from the MDSC-P library, 5,922,188 from the MDSC-D1 library and 5,935,963 from the MDSC-D3 library after deleting some contaminant reads (Table 1). Length distribution analysis showed that most reads ranged from 21 to $23 \mathrm{nt}$. The percentage of 22-nt reads in total reads was $72.76,71.26$, and $75.58 \%$ in the MDSC-P, MDSC-D1, and MDSC-D3 library, respectively (Fig. 2). The reads for the three libraries $(5,188,810,5,041,921$ and 5,236,492, respectively) were perfectly matched to the bovine genome (Table 2).

\section{Identification of conserved bovine miRNAs}

To identify conserved miRNAs in MDSCs during differentiation, the small RNAs with a length of 18-23 nucleotides were Blastn searched against miRBase 21.0 (miRBase release version V21.0, July 3, 2014). The miRNA candidates were then clustered into 439, 394, and 392 categories corresponding to 455, 412, and 410 independent genomic loci in the three libraries according to sequence similarity (Table 3), of which 322 miRNAs overlapped in three libraries (Additional file 2).

The expression of known miRNAs was demonstrated by generating $\log _{2}$ ratio plots and scatter plots (Fig. 3). The expression profiles between the different libraries are shown in Additional file 3. The results showed that 296 miRNAs comprised 9 upexpressed (Table 4), 165 down-expressed, and 122 equally expressed miRNAs in MDSC-D1 compared to those in MDSC-P; 304 miRNAs comprised 15 up-expressed (Table 4), 145 down-expressed, and 144 equally expressed miRNAs in MDSC-D3 compared to those in MDSC-P; 273 miRNAs comprised 17 up-expressed, 55 downexpressed, and 201 equally expressed miRNAs in MDSC-D3 compared to those in

Table 1 Summary of small RNA sequencing data

\begin{tabular}{|c|c|c|c|c|c|c|}
\hline \multirow[t]{2}{*}{ Type } & \multicolumn{2}{|l|}{ MDSC-P } & \multicolumn{2}{|l|}{ MDSC-D1 } & \multicolumn{2}{|c|}{ MDSC-D3 } \\
\hline & Count & $\%$ & Count & $\%$ & Count & $\%$ \\
\hline total_reads & 6000000 & & 6000000 & & 6000000 & \\
\hline high_quality & 5984373 & $100 \%$ & 5984335 & $100 \%$ & 5984569 & $100 \%$ \\
\hline 3'adaptor_null & 2916 & $0.05 \%$ & 2967 & $0.05 \%$ & 2901 & $0.05 \%$ \\
\hline insert_null & 3115 & $0.05 \%$ & 1252 & $0.02 \%$ & 1408 & $0.02 \%$ \\
\hline 5'adaptor_contaminants & 86464 & $1.44 \%$ & 54566 & $0.91 \%$ & 39651 & $0.66 \%$ \\
\hline smaller_than_18nt & 20713 & $0.35 \%$ & 3297 & $0.06 \%$ & 4615 & $0.08 \%$ \\
\hline polyA & 110 & $0.00 \%$ & 65 & $0.00 \%$ & 31 & $0.00 \%$ \\
\hline clean_reads & 5871055 & $98.11 \%$ & 5922188 & $98.96 \%$ & 5935963 & $99.19 \%$ \\
\hline
\end{tabular}

Note: total_reads: total sequenced reads, which is required to be greater than $5 \mathrm{M}$ in general; high_quality: number of high quality reads with no N, no more than 4 bases whose quality score was lower than 10 and no more than 6 bases whose quality score was lower than 13; 3'adaptor_null: number of reads with no 3'adaptor; insert_null: number of reads with no insertion; 5'adaptor_contaminants: number of 5'contaminants; smaller_than_18nt: number of reads less than $18 \mathrm{nt}$ generally. Small RNA tags were between 18 and $30 \mathrm{nt}$ long, so too short tags should be removed from data for further analysis; polyA: number of reads with polyA; clean_reads: number of clean reads after adaptors and contaminants were removed, which were used in the following analysis 


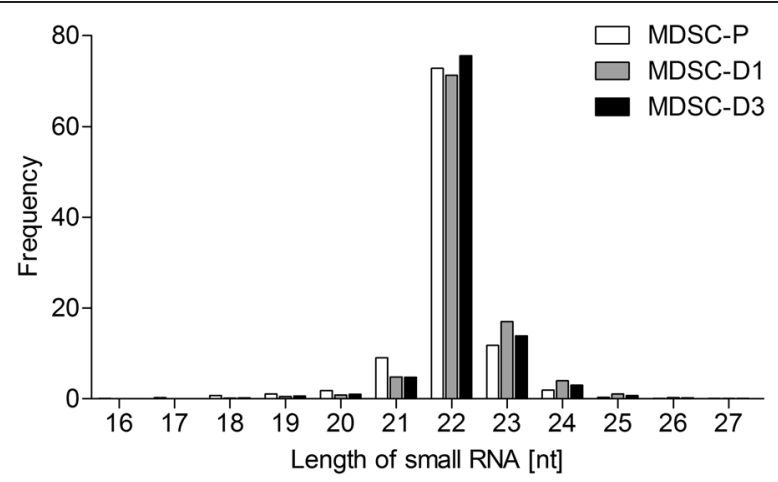

Fig. 2 Length distributions of small RNAs in the three RNA libraries. White columns represent length distributions of small RNAs in the MDSC-P library; gray columns represent length distributions of small RNAs in the MDSC-D1 library; black columns represent length distributions of small RNAs in the MDSC-D3 library

MDSC-D1. Additional file 4: Table S1 lists the 10 most abundant miRNAs in MDSC-P, MDSC-D1, and MDSC-D3. The miRNA with the greatest count in MDSC-D3 was miR-206, a major miRNA in skeletal muscle development, with an average normalized read count of $>1$ million. The ubiquitously expressed let-7 members, let-7a-5p, let-7f, and let-7b, followed, and miR-1 had the fifth greatest count. The miRNA expression patterns during MDSC differentiation were clustered using hierarchical cluster analysis (Additional file 5: Figure S1). For example, compared with the proliferation stages (MDSC-P), the expression levels of miR-2443, miR-423-5p, miR-181a, miR-10a, and miR-206 were higher in MDSC-D1, and the pattern was the same as that of miR-139, miR-1, miR-95, miR-206, and miR-133a in MDSC-D3.

Nucleotide bias analysis at each position showed that GC content was high at the 2nd, 11th, and 15th positions with values of $94.34,92.53$, and $95.16 \%$, respectively, but not at the 1st, 6th, 9th, 10th, 13th, 14th, 16th, 22nd, and 24th positions with values of $0.70,6.69,4.94,5.91,4.69,3.69,3.40,7.17$, and $0.18 \%$, respectively, in the MDSC-P library. A similar result was obtained in the MDSC-D1 and MDSC-D3 libraries. In all libraries, nucleotides $\mathrm{A}+\mathrm{U}$ were distributed mainly in the remaining positions with the exception of the 4th, 5th, 8th, 12th, 20th, and 23rd positions (Additional file 6: Figure S2). The phenomenon of nucleotide bias might be related to the mechanisms of miRNA, such as binding with targets for gene regulation. The 1st, 9th, and terminal positions were enriched with $U$ and the 1st and 9th positions were the limits of the "seed region" of a miRNA which was responsible for targeting mRNAs for gene regulation [21]. A similar result was obtained in MDSC-P miRNAs at the 1st, 9th, and end positions, but there was only $44.35 \% \mathrm{U}$ at the end position in MDSC-D1 and $58.21 \%$ at the 9th position in the MDSC-D3 library. The differences observed between our study and previous studies might have been due to different experimental approaches or differences in samples [12].

Positions 2-8 of a mature miRNA are called the seed region, which are highly conserved. The target of a miRNA might differ with changes of nucleotides in this region. In our study, miRNAs that might have a base edit could be detected by aligning unannotated sRNA tags with mature miRNAs from miRBase21, allowing one mismatch at a given position. The results showed that the mismatches occurred in all three libraries with the percentage of $22.65,22.45$, and $23.53 \%$, respectively. The mismatches could 
Table 2 Distribution of the genome-mapped sequence reads in small RNA libraries

\begin{tabular}{|c|c|c|c|c|c|c|}
\hline \multirow[t]{2}{*}{ Locus class } & \multicolumn{2}{|l|}{ MDSC-P } & \multicolumn{2}{|l|}{ MDSC-D1 } & \multicolumn{2}{|l|}{ MDSC-D3 } \\
\hline & Unique sRNAs & Total sRNAs & Unique sRNAs & Total sRNAs & Unique sRNAs & Total sRNAs \\
\hline Total & $106806(100 \%)$ & $5871055(100 \%)$ & $68492(100 \%)$ & $5922188(100 \%)$ & $66603(100 \%)$ & $5935963(100 \%)$ \\
\hline miRNA & 3056 (2.86 \%) & 4844028 (82.51%) & 2328 (3.40 \%) & $4850321(81.90 \%)$ & 2483 (3.73 \%) & $5051419(85.10 \%)$ \\
\hline exon_antisense & $473(0.44 \%)$ & 1428 (0.02 \%) & $164(0.24 \%)$ & 410 (0.01\%) & $141(0.21 \%)$ & $467(0.01 \%)$ \\
\hline exon_sense & 5111 (4.79 \%) & 7313 (0.12 \%) & 3167 (4.62 \%) & $4056(0.07 \%)$ & 2892 (4.34 \%) & 3564 (0.06 \%) \\
\hline intron_antisense & 2759 (2.58 \%) & 4632 (0.08 \%) & 899 (1.31 \%) & 1355 (0.02 \%) & 874 (1.31 \%) & $1201(0.02 \%)$ \\
\hline intron_sense & 4057 (3.80 \%) & $7573(0.13 \%)$ & 1823 (2.66 \%) & $2844(0.05 \%)$ & 1337 (2.01 \%) & $2175(0.04 \%)$ \\
\hline rRNA & $9082(8.50 \%)$ & $64109(1.09 \%)$ & $9408(13.74 \%)$ & $79061(1.33 \%)$ & $9454(14.19 \%)$ & 108407 (1.83 \%) \\
\hline repeat & 7322 (6.86 \%) & 15735 (0.27 \%) & 2490 (3.64 \%) & 4508 (0.08 \%) & 2208 (3.32 \%) & 4167 (0.07 \%) \\
\hline scRNA & $104(0.10 \%)$ & 1025 (0.02 \%) & $120(0.18 \%)$ & $2064(0.03 \%)$ & $134(0.20 \%)$ & 2295 (0.04 \%) \\
\hline snRNA & $591(0.55 \%)$ & 2554 (0.04 \%) & $461(0.67 \%)$ & 2024 (0.03 \%) & $332(0.50 \%)$ & 1454 (0.02 \%) \\
\hline snoRNA & $583(0.55 \%)$ & 1666 (0.03 \%) & $568(0.83 \%)$ & 1496 (0.03 \%) & 487 (0.73 \%) & 1374 (0.02 \%) \\
\hline srpRNA & $66(0.06 \%)$ & $134(0.00 \%)$ & $100(0.15 \%)$ & 175 (0.00 \%) & $85(0.13 \%)$ & $312(0.01 \%)$ \\
\hline tRNA & 5712 (5.35 \%) & 48058 (0.82 \%) & 3893 (5.68 \%) & 29959 (0.51\%) & 4428 (6.65 \%) & 35856 (0.60 \%) \\
\hline unann & 67890 (63.56 \%) & 872800 (14.87 \%) & 43071 (62.88 \%) & 943915 (15.94 \%) & 41748 (62.68 \%) & 723272 (12.18 \%) \\
\hline
\end{tabular}


Table 3 Summary of known miRNA in RNA libraries

\begin{tabular}{llllllll}
\hline & miR & miR* $^{*}$ & miR-5p & miR-3p & pre-miRs & $\begin{array}{l}\text { Unique matched to } \\
\text { pre-miRs }\end{array}$ & $\begin{array}{l}\text { Read matched to } \\
\text { pre-miRs }\end{array}$ \\
\hline Known miRs & 598 & 0 & 79 & 78 & 766 & - & - \\
MDSC-P & 339 & 0 & 51 & 49 & 455 & 3081 & 4844156 \\
MDSC-D1 & 297 & 0 & 49 & 48 & 412 & 2352 & 4850401 \\
MDSC-D3 & 297 & 0 & 46 & 49 & 410 & 2501 & 5051499 \\
\hline
\end{tabular}

Note: * means minor miR sequences

be caused by post-transcriptional modification, and/or RT-PCR, and sequencing errors (Additional file 7).

In our study, further analysis identified a total of 439, 394, and 392 conserved miRNAs that belonged to 237 miRNA families in the three libraries mentioned above. The largest miRNA family identified was miR-2284, which consisted of 63 members, and miR-154, let-7, and miR-181/30 possessed 18, 12, and 6 members, respectively; other miRNA families, such as miR-122, miR-1249, miR-140, and miR-486, had only one member, whereas miR-1940, miR-2286, miR-3431, and miR-574 did not belong to any gene family (Additional file 8 ).

\section{Identification of novel bovine miRNAs}

The characteristic hairpin structure of a miRNA precursor could be used to predict novel miRNAs. The prediction software Mireap was developed to predict novel miRNAs by exploring the secondary structure, the Dicer cleavage site, and the minimum free energy of the unannotated small RNA reads that could be mapped to a genome. Based on HiSeq deep sequencing, 53 novel bovine miRNAs were identified in bovine MDSCs, which corresponded to 145 genomic loci. Forty-three novel miRNAs were in the MDSC-P library, 17 were in the MDSC-D1, and 19 were in the MDSC-D3 library, and 26 of the miRNAs overlapped in three libraries (Additional file 9). The read numbers from HiSeq deep sequencing are often regarded as a reliable quantification of miRNA expression; the read numbers of miRNAs in the HiSeq deep sequencing analysis are shown in Additional file 10.
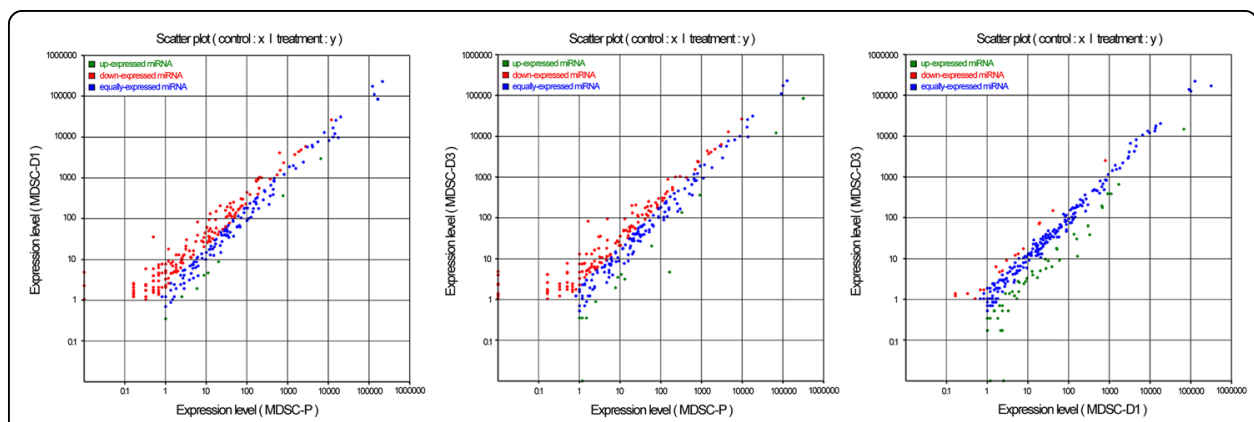

Fig. 3 Differential expression of conserved miRNAs during differentiation of MDSCs. Compare the known miRNA expression between different differentiation stages to find out the differentially expressed miRNA. Each point in the figure represents a miRNA. The $X$ axis and $Y$ axis show the expression level of miRNAs in two libraries. Green points represent miRNAs with ratio $>2$; Blue points represent miRNAs with $1 / 2<$ ratio $\leq 2$; Red points represent miRNAs with ratio $\leq 1 / 2$. Ratio $=$ Normalized expression in treatment/Normalized expression in control 
Table 4 miRNAs highly expressed in MDSC-D1 and MDSC-D3 compared with MDSC-P

\begin{tabular}{|c|c|c|c|c|c|}
\hline Category & miRNA & MDSC-P & MDSC-D1 & MDSC-D3 & $\begin{array}{l}\text { log2 Ratio } \\
\text { (Fold Change) }\end{array}$ \\
\hline \multirow[t]{8}{*}{ MDSC-D1/MDSC-P } & bta-miR-99a-3p & 11 & 35 & & 1.66 \\
\hline & bta-miR-2396 & 2 & 6 & & 1.57 \\
\hline & bta-miR-139 & 27 & 67 & & 1.29 \\
\hline & bta-miR-2443 & 51 & 121 & & 1.23 \\
\hline & bta-miR-423-5p & 16887 & 38687 & & 1.18 \\
\hline & bta-miR-181a & 2105 & 4694 & & 1.14 \\
\hline & bta-miR-10a & 24 & 53 & & 1.13 \\
\hline & bta-miR-2331-5p & 7 & 15 & & 1.08 \\
\hline \multirow[t]{15}{*}{ MDSC-D3/MDSC-P } & bta-miR-2904 & 0 & & 7 & 6.88 \\
\hline & bta-miR-139 & 27 & & 990 & 5.18 \\
\hline & bta-miR-1 & 69315 & & 408668 & 2.54 \\
\hline & bta-miR-2411-5p & 2 & & 9 & 2.15 \\
\hline & bta-miR-95 & 18 & & 78 & 2.09 \\
\hline & bta-miR-99a-3p & 11 & & 46 & 2.04 \\
\hline & bta-miR-206 & 493002 & & 1890636 & 1.92 \\
\hline & bta-miR-2396 & 2 & & 7 & 1.79 \\
\hline & bta-miR-421 & 119 & & 361 & 1.58 \\
\hline & bta-miR-500 & 5 & & 15 & 1.56 \\
\hline & bta-miR-874 & 2 & & 6 & 1.56 \\
\hline & bta-miR-10a & 24 & & 64 & 1.39 \\
\hline & bta-miR-503-5p & 2066 & & 5503 & 1.39 \\
\hline & bta-miR-362-5p & 20 & & 53 & 1.39 \\
\hline & bta-miR-133a & 774 & & 1994 & 1.34 \\
\hline
\end{tabular}

Note: Fold Change $=$ MDSC-D1 $/$ MDSC - P; Fold Change $=$ MDSC-D3 $/$ MDSC-P

The expression of novel miRNAs in MDSC-P, MDSC-D1, and MDSC-D3 was demonstrated by generating $\log _{2}$ ratio plots and scatter plots (Additional file 11: Figure S3). The results showed that 42 miRNAs comprised 7 up-expressed, 31 down-expressed, and 4 equally expressed miRNAs in MDSC-D1 compared to those in MDSC-P; 43 miRNAs comprised 8 up-expressed, 32 down-expressed, and 3 equally expressed miRNAs in MDSC-D3 compared to those in MDSC-P; and 24 miRNAs comprised 9 up-expressed, 9 down-expressed, and 6 equally expressed miRNAs in MDSC-D3 compared to those in MDSC-D1.

\section{qPCR analysis confirmed differential expression of selected miRNAs in MDSCs}

To confirm the RNA-seq results, the expression of 12 miRNAs was quantified by stemloop qPCR [22]. The results showed that miR-29a, miR-27a, and let-7i were highly expressed in MDSC-P; in contrast, miR-320, miR-1, and miR-206 were highly expressed in MDSC-D3. In addition, miR-206, miR-1, and miR-320 were up-regulated during MDSC differentiation; miR-495, miR-133b, and miR-487 were down-regulated in MDSC-D1 and upregulated in MDSC-D3. The results of the RT-qPCR analysis were consistent with those obtained by RNA-seq analysis except for miR-423, which was upregulated in MDSC-D3 compared with MDSC-D1 in RT-qPCR, while it was down- 
regulated in RNA-seq (Fig. 4). These results indicated that the HiSeq deep sequencing in our study was of high reliability.

\section{Target prediction for miRNAs}

The function of a miRNA is ultimately defined by the genes it targets and by its effect on the expression of these genes. To identify the potential targets of miRNAs, we searched a bovine mRNA (ftp://hgdownload.cse.ucsc.edu/goldenPath/bosTau7/database/refGene.txt.gz) database. Table 5 lists the target genes obtained from MDSC-P, MDSC-D1, and MDSC-D3, which might be regulated by the miRNAs in MDSC-D1, MDSC-D3 and MDSC-P, shown in Additional file 12. The binding energy of the conserved miRNAs with their targets varied from -11.2 to $-44.3 \mathrm{kcal} / \mathrm{mol}$ and from 10.3 to $-49.4 \mathrm{kcal} / \mathrm{mol}$ between the novel miRNAs and their targets (Additional file 13). Some miRNAs had more than 1000 predicted targets, and some other target genes were putatively regulated by more than two miRNAs.

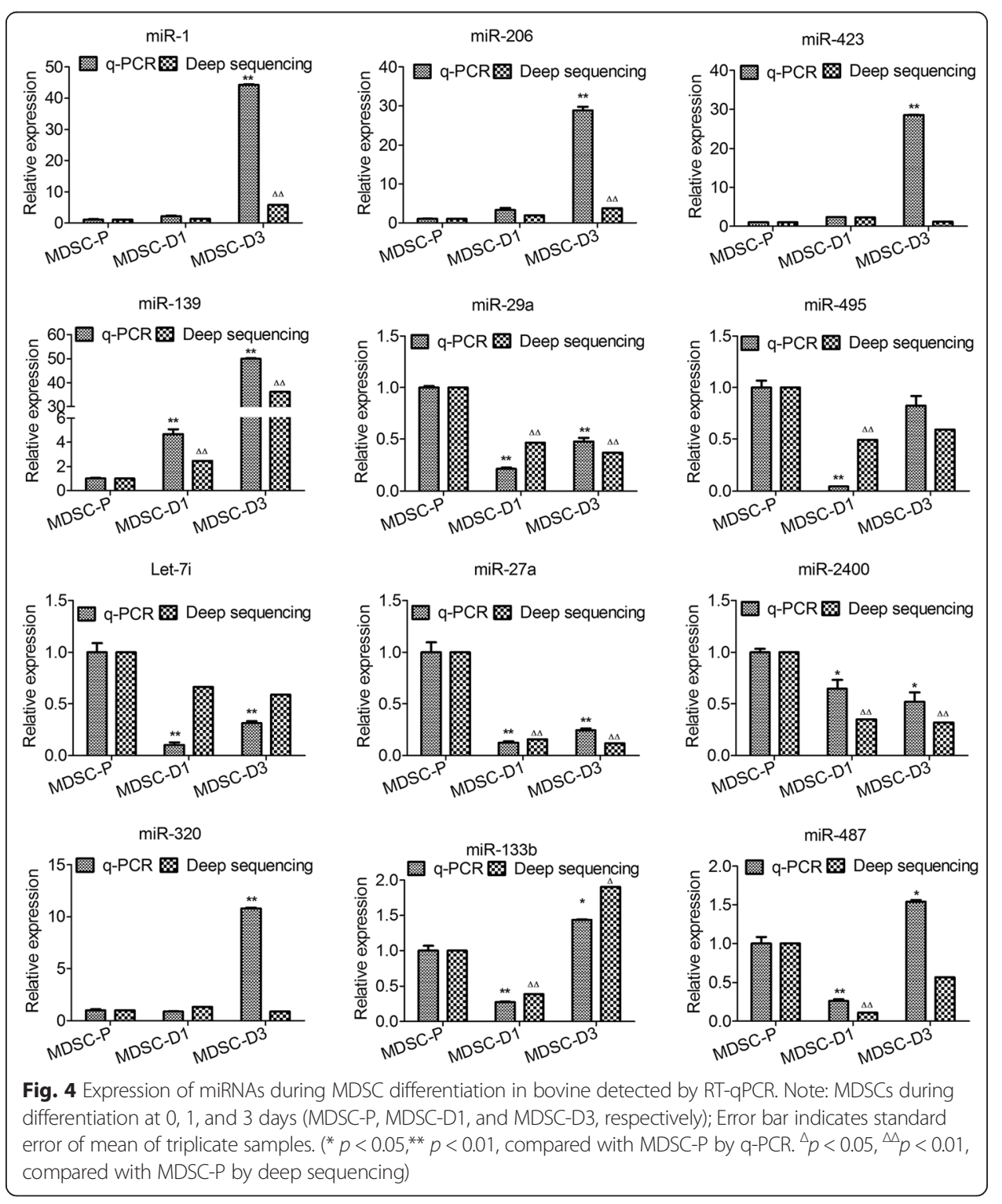


Table 5 Summary of known miRNA and novel miRNA target prediction

\begin{tabular}{llll}
\hline & sample & miRNA number & Target number \\
\hline known miRNA & MDSC-P & 439 & 962,279 \\
& MDSC-D1 & 394 & 857,309 \\
novel miRNA & MDSC-D3 & 392 & 861,755 \\
& MDSC-P & 42 & 87,921 \\
& MDSC-D1 & 17 & 41,798 \\
& MDSC-D3 & 20 & 43,423 \\
\hline
\end{tabular}

\section{GO enrichment analysis and KEGG pathway analysis of target genes}

To understand the biological function of miRNAs in MDSCs, all the predicted target genes were classified according to KEGG functional annotations, which help to identify pathways that were actively regulated by miRNAs in MDSCs. Most of these genes were involved in cellular metabolism, pathways in cancer, actin cytoskeleton regulation and the MAPK signaling pathway (Table 6). The most commonly indicated pathway was the metabolic pathway, with 1321 genes representing $12.44 \%$ of the total, followed by the pathways in cancer (3.51\%), biosynthesis of secondary metabolites (3.46\%), regulation of actin cytoskeleton $(3.17 \%)$ and the MAPK signaling pathway (3.03\%). All the predicted target genes were submitted for Gene Ontology (GO) analysis using an online version of the Blast2GO program (www.Blast2GO.com). In total, 10,887 genes were termed good or better than 1 using the component ontology with $\mathrm{P}$ value analysis, 10,228 genes were assigned different functions and 10,039 genes were termed on biological processes (Additional file 4: Table S2). To determine the potential functions of the different expression miRNAs, the 45 most abundant miRNAs in the three libraries were selected for Gene Ontology analysis. The main GO categories targeted by different expression genes included developmental process, cell death, growth, reproductive process, and metabolic process (Fig. 5). Further analysis for miRNA targets is needed and will help us to gain insight into the roles of these miRNAs in MDSC differentiation.

Table 6 The 10 most-enriched KEGG pathways for the target genes of known miRNAs

\begin{tabular}{llll}
\hline Pathway & $\begin{array}{l}\text { Target genes with } \\
\text { pathway annotation }\end{array}$ & P-value & Pathway ID \\
\hline Metabolic pathways & $1321(12.44 \%)$ & 0.4507364 & ko01100 \\
Pathways in cancer & $373(3.51 \%)$ & 0.8069502 & ko05200 \\
Biosynthesis of secondary metabolites & $367(3.46 \%)$ & 0.8097889 & ko01110 \\
Regulation of actin cytoskeleton & $337(3.17 \%)$ & 0.8241077 & ko04810 \\
MAPK signaling pathway & $322(3.03 \%)$ & 0.8313458 & ko04010 \\
HTLV-I infection & $297(2.8 \%)$ & 0.8435271 & ko05166 \\
Endocytosis & $292(2.75 \%)$ & 0.8459811 & k004144 \\
Focal adhesion & $285(2.68 \%)$ & 0.8494267 & ko04510 \\
Tight junction & $266(2.51 \%)$ & 0.8588381 & k004530 \\
Tuberculosis & $262(2.47 \%)$ & 0.8608305 & ko05152 \\
Phagosome & $251(2.36 \%)$ & 0.8663294 & k004145 \\
Epstein-Barr virus infection & $244(2.3 \%)$ & 0.869844 & ko05169 \\
Neuroactive ligand-receptor interaction & $239(2.25 \%)$ & 0.8723616 & ko04080 \\
Cytokine-cytokine receptor interaction & $237(2.23 \%)$ & 0.8733703 & ko04060 \\
Influenza A & $236(2.22 \%)$ & 0.873875 & ko05164 \\
\hline
\end{tabular}




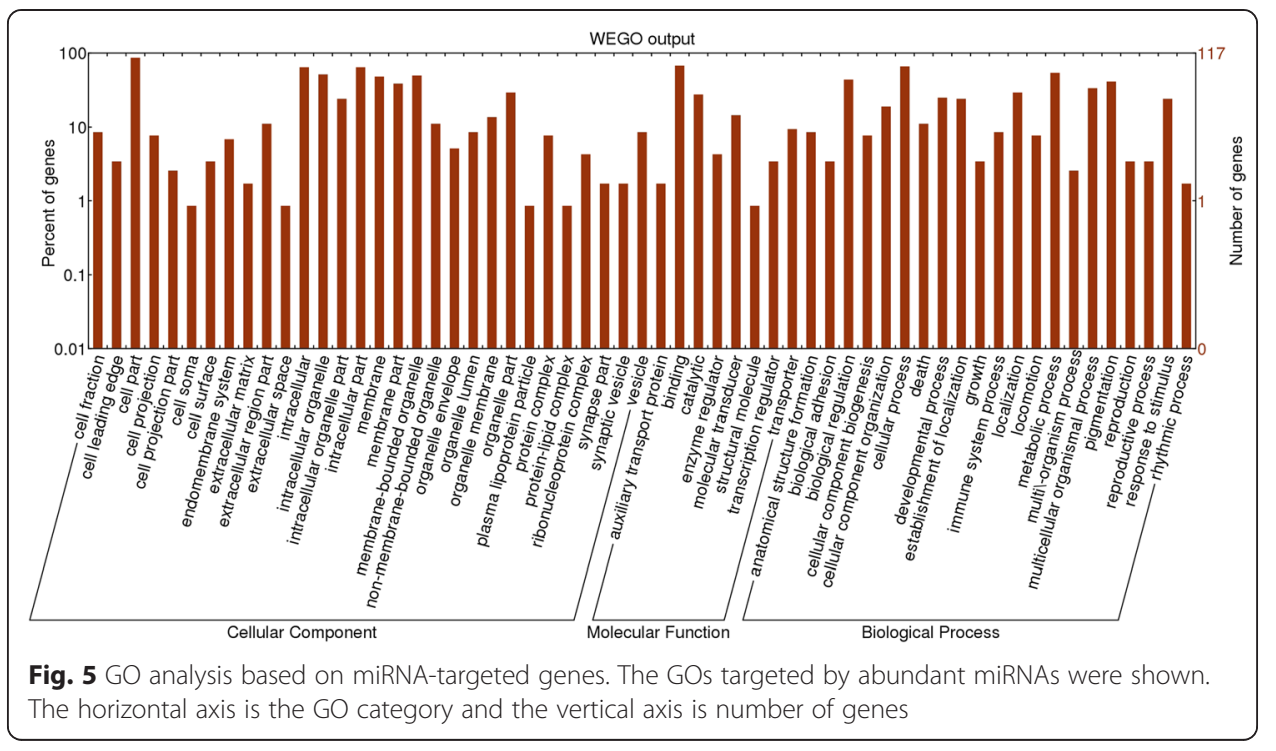

\section{Discussion}

A series of regulatory changes in gene expression occur during skeletal muscle growth and development $[3,5,23,24]$, and decipherment of these changes is essential for the production of high-quality meat products [25]. The miRNAs are a class of gene expression regulatory factors that play critical roles in muscle cell differentiation [26-29], and identifying the differentially expressed miRNAs is an important first step to investigating the function of miRNAs in the course of MDSC differentiation by high throughput sequencing [30]. In this study we used a next-generation sequencing platform to determine the identities and expression levels of different miRNAs involved in muscle development in an in vitro cell culture system. To investigate microRNAs reliably, we collected 9 plates of cells (isolated from hind-limb muscles of 3 newborn Chinese Simmental calves) from each of the MDSCs in different stages of differentiation. Total RNA was extracted and pooled to identify known and novel miRNAs. In total, 617 miRNAs, including 53 novel miRNA candidates, were identified, and 322 miRNAs were common to all three stages, whereas 117 miRNAs only occurred in MDSC-P, 72 miRNAs only in MDSC-D1, and 70 miRNAs only in MDSC-D3 (Additional file 2).

All conserved miRNAs which were identified in the three libraries belonged to 237 miRNA families. The identified miRNA families have been shown to be conserved in a variety of species. For example, the let-7, miR-25, miR-1, miR-10, miR-8, miR-9, and miR-124 families have been found in 68, 68, 69, 70, 71, 72, and 73 species, respectively, while the miR-2484, miR-2300, miR-2319, miR-2329, miR-2363, miR-2404, miR-2450, miR-2887, miR-3432, miR-2604, miR-6526, and miR-6536 families have only been detected in cattle (Additional file 8 ). The miRNA family analysis might suggest a speciesspecific expression profile of miRNAs.

The finding that most members of conserved miRNA families were expressed in MDSCs supported the idea that regulatory or functional diversification occurred [31, 32]. Different family members also displayed drastically different expression levels. For example, the abundance of the miR-2284 family varied from 1 read (bta-miR-2284 m, bta-miR-2284n, bta-miR-2285c) to 34,204 reads (bta-miR-2284x) with RNA-seq. This was also the case for some other miRNA families, such as bta-let-7 (1442-1,331,479 
reads), bta-miR-10 (24-10,950 reads), bta-miR-29 (1-153,254 reads) and bta-miR-181 (1-4694 reads). The expression levels of some miRNA families were similar, such as miR-1271, in which 12, 5, and 2 reads were detected, respectively. The existence of a dominant member in a miRNA family might suggest that the regulatory role of this family was performed by the dominant member at the developmental time when the samples were collected for RNA extraction. Abundance comparisons of different members of a miRNA family might provide valuable information on the role that miRNAs play in that specific stage of MDSCs.

Recent studies showed that myogenic differentiation was regulated by muscle-specific miRNA. miR-1 and miR-206 were upregulated during satellite cell differentiation and promoted myogenesis $[28,33]$, whereas miR-133 was involved primarily in the promotion of proliferation [9]. In this study, sequence comparison with known miRNAs identified the muscle-specific miR-206 as the most abundant miRNA across all MDSC samples, which represented more than $37.39 \%$ of all miRNAs in MDSC-D3, and represented $10.15 \%$ abundance in proliferating satellite cells. The constant high-level expression at all stages after early differentiation suggested that the role of miR-206 was to repress functions associated with muscle precursor cells. Combined with the facts that miR-206 was lowly abundant in proliferating cells of mouse $\mathrm{C} 2 \mathrm{C} 12$ cells, and was reported to be induced during differentiation [34], we proposed that its presence was associated with the switch from precursor to mature muscle cell. Compared with the high abundance of miR-206 in MDSCs, bovine miR-1 showed moderate abundance, and then increased throughout the differentiation. The expression pattern was similar to the one observed for this miRNA in mouse muscle development [9]. These data suggested that miR-1 plays different roles from miR-206 in muscle differentiation; miR-1 could affect the regulation of genes that require inactivation in later stages, while miR-206 could have a more constant role in repressing genes immediately after differentiation. Interestingly, miR-133 was detected at low levels in MDSC-D1, but higher in MDSC-D3. Based on abundance levels, miR-206 and miR-1 were considered to have a greater role in MDSC differentiation than miR-133 or their targets could be more abundant. In addition, both miR-206 and miR-1 promoted differentiation [9], which suggested that these two miRNAs might have a greater effect than miR-133 in muscle satellite cell differentiation [35].

In addition, the miR-27b expression level was 1.43- and 1.48-fold lower in MDSC-D1 and MDSC-D3, respectively, than in MDSC-P. The expression of miR-27b was consistent with the previous report of reduced myostatin expression through targeting the 3'UTR, resulting in myoblast proliferation [36]. On the other hand, miR-27b was reported to be highly expressed in mouse satellite cells after differentiation [11]. Thus, the molecular function of miR-27b might be divergent, depending on the type of muscles, as well as animal species.

In addition to muscle-specific miRNAs, a larger number of ubiquitous miRNAs were present in all libraries. Bta-miR-99a-3p, bta-miR-2396, bta-miR-139, and bta-miR-10a presented at 2-fold greater abundance in MDSCs than in MDSC-P (Table 4). These miRNAs were implicated in multiple cellular processes, including inhibition of growth (miR-99a and miR-139), induction of apoptosis (miR-99a and miR-139), cell cycle arrest (miR-99a), and promotion of differentiation (miR-10a) [37-41]. In addition, miR-181a was up-regulated in MDSC-D1, which promoted apoptosis by targeting Bcl-2 [42] and 
suppressed tumor growth by targeting the MAPK-Snai2 pathway [43]. miR-503 $\left(\log _{2}\right.$ $($ MDSC-D3/MDSC-P $)=1.39)$ suppressed cell proliferation and cell cycle progression, and promoted cell cycle quiescence by targeting cyclin D1 [44]. Conversely, 143 miRNAs presented at 2-fold lower abundance in MDSC-D1 than in MDSC-P; for example, miR-184 $\left(\log _{2}(\right.$ MDSC-D3/ MDSC-P $\left.)=-2.29\right)$ promoted cell proliferation by targeting c-Myc [45]. Nevertheless, the role of these miRNAs during differentiation of MDSCs is yet to be fully determined.

In addition to the 564 known miRNAs, we also discovered 53 novel bovine miRNAs. Sixteen of the novel miRNAs were found to be conserved among mammals, including rat, mouse, human, rhesus monkey, chimpanzee, pig and dog. The 37 novel bovine miRNAs had not been identified in any other species and may be specific to cattle or skeletal muscle. The novel miRNAs of bta-n25 were relatively abundant in MDSC-P and MDSC-D3, and therefore might be involved in MDSC differentiation.

The results of GO term analysis showed further comprehensive biological processes that miRNA regulated in MDSC differentiation. In total, 10,228 genes were assigned different functions and 10,039 genes were classified into biological processes; this might be because the target genes were predicted. The distributions of GO term categories were similar for the target genes of miRNAs in MDSC-P, MDSC-D1 and MDSC-D3. The biological functions identified by the Blast2GO program included categories related to a wide variety of physiological and biological events, such as cellular developmental processes, metabolic processes, biological regulation and cell differentiation. Further analysis is needed about miRNA target genes which are regulated by musclespecific miRNAs and would help us to gain insight into the roles of these miRNAs in MDSC differentiation.

Skeletal muscle is known to be one of the major tissues accounting for energy expenditure; both proliferating and differentiating cells must rapidly generate new biomass in the form of nucleotides, proteins and phospholipids to support rapid cell division and growth. The miRNAs regulate a variety of physiologic processes, including glucose and lipid metabolism [46, 47]. To date, several miRNAs have been reported to regulate lipid metabolism, including miR-122, miR-33a, miR-133, miR-335, miR-125a, and miR-34a [48]. These miRNAs were down-regulated in MDSC-D1 in our study. miR-33 regulated genes associated with $\beta$-oxidation of fatty acids, including HADHB, CROT and CPT1A [49]. Several studies have demonstrated that miR-122 plays a regulatory role in lipid metabolism. Transfection of an anti-sense oligonucleotide inhibitor of mmu-miR-122 into a mouse hepatocyte-derived cell line (AML12) caused an increase of mRNA of six genes, ie GYS1, SLC7A1, MINK1, ALDOA, CCNG1 and P4HA1 [50], some of which might have indirect effects on lipid metabolism. The miR-335 expression level was closely correlated with expression levels of adipocyte differentiation markers such as PPAR $\gamma$, aP2, and FAS in 3 T3-L1 adipocytes [51]. Moreover, forced expression of miR-133 decreased GLUT4 expression and reduced insulin-mediated glucose uptake in cardiomyocytes [52]. Overexpression of miR-29a-c in primary hepatocytes and mouse livers decreased the protein levels of PGC-1a and G6 Pase [53]. MiR-195-5p decreased T24 cell glucose uptake, inhibited cell growth and promoted cell apoptosis through suppression of GLUT3 expression [54]. Ectopic expression of miR-27 inhibited the expression of PPAR $\gamma$ and C/EBPo in 3 T3-L1 cells [55]. MiRNA expression patterns may differ by tissue type, suggesting that this mechanism 
of gene expression regulation was dynamic and may be highly specific, indicating the need for further study to determine their importance in MDSC differentiation.

\section{Conclusions}

This study confirmed the authenticity of 564 known miRNAs, and discovered 53 novel miRNAs in MDSCs during differentiation using RNA-seq. This study expanded the repertoire of bovine miRNAs and could initiate further research in bovine muscle differentiation. In addition, our results revealed distinct miRNA expression patterns during the time course of MDSC proliferation and differentiation, cell cycle progression, cell apoptosis and metabolic pathways. These results would warrant further investigation to determine the regulatory roles of the differentially expressed miRNAs identified in the present study in controlling muscle differentiation.

\section{Additional files}

Additional file 1: Primers and sequences for RT-qPCR analysis (XLS $12 \mathrm{~kb}$ )

Additional file 2: Bovine conserved miRNAs. Note: The data in this supplementary file can be seen in the Excel spreadsheet. (XLS $273 \mathrm{~kb}$ )

Additional file 3: The known miRNA expression profiles between two libraries. Note: The data in this supplementary file can be seen in the Excel spreadsheet.

Additional file 4: Table S1. Top 10 most frequently detected known miRNA in MDSCs. Suppl. Table S2. The 10 most-enriched GO categories for the target genes of known miRNAs. (DOC $83 \mathrm{~kb}$ )

Additional file 5: Figure S1. Hierarchical cluster analysis of differentially expressed miRNAs during MDSC differentiation. Cluster analysis was performed for differentially expressed miRNAs after data adjustment (log transformation, median center, and normalization). The color codes of green, black, and red represent high, average, and low expression levels, respectively. Note: each row in the figure shows one miRNA, and each column shows one sample pair. So each cell shows the differential expression of a miRNA in one sample pair. In cluster analysis miRNAs that have a similar pattern of differential expression in different sample pairs were clustered together. (JPG $2732 \mathrm{~kb}$ )

Additional file 6: Figure S2. Nucleotide bias at each position of sRNA tags. Note: miRNA nucleotide bias at each position of MDSC-P (A), MDSC-D1 (B) and MDSC-D3 (C), respectively. Note: each color in the figure shows the sRNA tags whose certain position was a certain base. (JPG $1036 \mathrm{~kb}$ )

Additional file 7: Summary of base edit in three libraries. Note: The data in this supplementary file can be seen in the Excel spreadsheet.

Additional file 8: Family analyses of known miRNAs. Note: The data in this supplementary file can be seen in the Excel spreadsheet.

Additional file 9: Novel miRNAs identified in this study. Note: The data in this supplementary file can be seen in the Excel spreadsheet.

Additional file 10: The novel miRNA expression profiles between two libraries. Note: The data in this supplementary file can be seen in the Excel spreadsheet.

Additional file 11: Figure S3. The differential expression of bovine novel miRNAs during different differentiation stages is shown. Compare the novel miRNA expression between different differentiation stages to find out the differentially expressed miRNA. Each point in the figure represents a miRNA. The $X$ axis and $Y$ axis show the expression level of miRNAs in two libraries. Green points represent miRNAs with ratio $>2$; blue points represent miRNAs with $1 / 2<$ ratio $\leq 2$; red points represent miRNAs with ratio $\leq 1 / 2$. Ratio=Normalized expression in treatment/ Normalized expression in control. (JPG $401 \mathrm{~kb}$ )

Additional file 12: Predicted target genes for up-regulation miRNA. Note: The data in this supplementary file can be seen in the Excel spreadsheet.

Additional file 13: Predicted target genes for novel miRNA. Note: The data in this supplementary file can be seen in the Excel spreadsheet.

\section{Abbreviations}

3' UTRs, 3' untranslated regions; DM, differentiation medium; DMEM, Dulbecco's modified Eagle's medium; GM, growth medium; GO, gene ontology; KEGG, Kyoto Encyclopedia of Genes and Genomes; MDSCs, skeletal muscle-derived satellite cells; MFEs, minimal free energies; miRNAs, microRNAs; RT-qPCR, reverse transcription quantitative real-time PCR

\section{Acknowledgements}

This work was supported by the breeding program for high-quality new varieties of genetically modified bovines from the National Major Transgenic Project (grant number 2014ZX08007-002). 
Availability of supporting data

All data with raw reads in the manuscript were deposited in an open access repository of the NCBI Sequence Read Archive (SRA) with the BioSample accession numbers SRS512681, SRS512683, SRS512684; the BioProject ID was PRNNA231753. The study accession number was SRP 034443. Other supporting data are included as Supplementary files.

\section{Authors' contributions}

WWZ gathered samples, conceived the report, participated in its design, performed data analysis, interpreted results and drafted the manuscript. XFS and HLT contributed to gathering samples and participated in intellectual discussion. YHW contributed to gathering samples. SFL involved in RNA-Seq experiments and revising the manuscript critically. YQY and GPL involved in obtaining funds for the study, design of study, interpretation of data and critically revising the manuscript. All authors read and approved the final manuscript.

\section{Competing interests}

The authors declare that they have no competing interests.

\section{Author details}

${ }^{1}$ The Laboratory of Cell and Development, Northeast Agricultural University, Mucai Street 59, Xiangfang District, Harbin 150030, Heilongjiang, China. ${ }^{2}$ College of Life Sciences and Agriculture \& Forestry, Qiqihar University, Qiqihar, Heilongjiang 161006, China. ${ }^{3}$ The Key Laboratory of Mammal Reproductive Biology and Biotechnology Ministry of Education, Inner Mongolia University, Hohhot 010021, China.

Received: 14 June 2015 Accepted: 25 September 2015

Published online: 28 July 2016

\section{References}

1. Dhawan J, Rando TA. Stem cells in postnatal myogenesis: molecular mechanisms of satellite cell quiescence, activation and replenishment. Trends Cell Biol. 2005;15:666-73.

2. Rudnicki MA, Le GF, Mckinnell I, Kuang S. The molecular regulation of muscle stem cell function. Cold Spring Harb Symp Quant Biol. 2008;73:323-31.

3. Weintraub H. The MyoD family and myogenesis: Redundancy, networks, and thresholds. Cell. 1994;75:1241-4.

4. Lassar AB, Skapek SX, Novitch B. Regulatory mechanisms that coordinate skeletal muscle differentiation and cell cycle withdrawal. Curr Opin Cell Biol. 1994;6:788-94.

5. Sabourin LA, Rudnicki MA. The molecular regulation of myogenesis. Clin Genet. 2000;57:16-25.

6. Ambros $V$. The function of animal MicroRNAs. Nature. 2004;431:350-5.

7. Ge Y, Chen J. MicroRNAs in skeletal myogenesis. Cell Cycle. 2011;10:441-8.

8. Yang Y, Sun W, Wang R, Lei C, Zhou R, Tang Z, et al. Wnt antagonist, secreted frizzled-related protein 1, is involved in prenatal skeletal muscle development and is a target of miRNA-1/206 in pigs. BMC Mol Biol. 2015;16:1-11.

9. Chen JF, Mandel EM, Thomson JM, Wu Q, Callis TE, Hammond SM, et al. The role of microRNA-1 and microRNA-133 in skeletal muscle proliferation and differentiation. Nat Genet. 2006;38:228-33.

10. Sweetman D, Goljanek K, Rathjen T, Oustanina S, Braun T, Dalmay T, et al. Specific requirements of MRFs for the expression of muscle specific microRNAs, miR-1, miR-206 and miR-133. Dev Biol. 2008;321:491-9.

11. Crist CG, Montarras D, Pallafacchina G, Rocancourt D, Cumano A, Conway SJ, et al. Muscle stem cell behavior is modified by microRNA-27 regulation of Pax3 expression. Proc Natl Acad Sci U S A. 2009;106:13383-7.

12. Sun J, Li M, Li Z, Jing X, Lan X, Zhang C, et al. Identification and profiling of conserved and novel microRNAs from Chinese Qinchuan bovine longissimus thoracis. BMC Genomics. 2013;14:42.

13. Lee S, Shin HSShireman PK, Vasilaki A, Van RH, Csete ME. Glutathione-peroxidase-1 null muscle progenitor cells are globally defective. Free Radic Biol Med. 2006;41:1174-84.

14. Tong HL, Yin HY, Zhang WW, Hu Q, Li SF, Yan YQ, et al. Transcriptional profiling of bovine muscle-derived satellite cells during differentiation in vitro by high throughput RNA sequencing. Cell Mol Biol Lett. 2015;20:351-73.

15. Li R, Li Y, Kristiansen K, Wang J. SOAP: short oligonucleotide alignment program. Bioinformatics. 2008;24:713-4.

16. Fu LX, Si QH, Kai G, An LX, Yi YZ, Li N, et al. Computational identification of novel microRNAs and targets in Brassica napus. Febs Letters. 2007;581:1464-74.

17. Ambros V, Bartel B, Bartel DP, Burge CB, Carrington JC, Chen X, et al. A uniform system for microRNA annotation. RNA. 2003;9:277-9.

18. Allen E, Xie Z, Gustafson AM, Carrington JC. microRNA-Directed Phasing during Trans-Acting siRNA Biogenesis in Plants. Cell. 2005;121:207-21.

19. Schwab R, Palatnik JF, Riester M, Schommer C, Schmid M, Weigel D. Specific effects of microRNAs on the plant transcriptome. Dev Cell. 2005;8:517-27.

20. Salone V, Rederstorff M. Stem-Loop RT-PCR Based Quantification of Small Non-Coding RNAs. Methods Mol Biol. 2015;1296:103-8.

21. Zhang B, Stellwag EJ, Pan X. Large-scale genome analysis reveals unique features of microRNAs. Gene. 2009:443:100-9.

22. Wang X. A PCR-based platform for microRNA expression profiling studies. RNA. 2009;15:716-23.

23. Pownall ME, Gustafsson MK, Emerson Jr CP. Myogenic regulatory factors and the specification of muscle progenitors in vertebrate embryos. Annu Rev Cell Dev Biol. 2002;18:747-83.

24. Song S, Huo JL, Li DL, Yuan YY, Yuan F, Miao YW. Molecular cloning, sequence characterization, and gene expression profiling of a novel water buffalo (Bubalus bubalis) gene, AGPAT6. Genet Mol Res. 2013;12:4116-26.

25. Muroya S, Taniguchi M, Shibata M, Oe M, Ojima K, Nakajima I, et al. Profiling of differentially expressed microRNA and the bioinformatic target gene analyses in bovine fast- and slow-type muscles by massively parallel sequencing. J Anim Sci. 2013;91:90-103.

26. Mi L, Li Y, Zhang $Q$, Zhao C, Peng Y, Yang G, et al. MicroRNA-139-5p regulates C2C12 cell myogenesis through blocking Wnt/ß-catenin signaling pathway. Biochem Cell Biol. 2015;93:1-8. 
27. Lei S, Bo Z, Li P, Schinckel AP, Liang T, Han W, et al. MicroRNA-128 targets myostatin at coding domain sequence to regulate myoblasts in skeletal muscle development. Cell Signal. 2015;27:1895-904.

28. Miretti S, Martignani E, Taulli R, Bersani F, Accornero P, Baratta M. Differential expression of microRNA-206 in skeletal muscle of female Piedmontese and Friesian cattle. Vet J. 2011;190:412-3.

29. Miretti S, Martignani E, Accornero P, Baratta M. Functional effect of mir-27b on myostatin expression: a relationship in Piedmontese cattle with double-muscled phenotype. BMC Genomics. 2013;14:1471-2164.

30. Jin W, Grant JR, Stothard P, Moore SS, Le LG. Characterization of bovine miRNAs by sequencing and bioinformatics analysis. BMC Mol Biol. 2009;10:2.

31. Liu N, et al. The evolution and functional diversification of animal microRNA genes. Cell Res. 2008;18:985-96

32. Cuperus JT, Fahlgren N, Carrington JC. Evolution and functional diversification of MIRNA genes. Plant Cell. 2011;23:431-42

33. Chen JF, Tao Y, Li J, Deng Z, Yan Z, Xiao X, et al. microRNA-1 and microRNA-206 regulate skeletal muscle satellite cell proliferation and differentiation by repressing Pax7. J Cell Biol. 2010;190:867-79.

34. Rao PK, Kumar RM, Farkhondeh M, Baskerville S, Lodish HF. Myogenic factors that regulate expression of muscle-specific microRNAs. Proc Natl Acad Sci U S A. 2006;103:8721-6.

35. Yin VP, Lepilina A, Smith A, Poss KD. Regulation of zebrafish heart regeneration by miR-133. Dev Biol. 2012;365:319-27.

36. Huang Z, Chen X, Bing Y, He J, Chen D. MicroRNA-27a promotes myoblast proliferation by targeting myostatin. Biochem Biophys Res Commun. 2012;423:265-9.

37. Cui L, Zhou H, Zhao H, Zhou Y, Xu R, Xu X, et al. MicroRNA-99a induces G1-phase cell cycle arrest and suppresses tumorigenicity in renal cell carcinoma. BMC Cancer. 2012;12:1552-6.

38. Chakrabarti M, Banik NL, Ray SK. Photofrin Based Photodynamic Therapy and miR-99a Transfection Inhibited FGFR3 and PI3K/Akt Signaling Mechanisms to Control Growth of Human Glioblastoma In Vitro and In Vivo. Plos One. 2013:8:e55652.

39. Jin $Y$, Tymen SD, Chen D, Fang ZJ, Zhao Y, Dragas D, et al. MicroRNA-99 family targets AKT/mTOR signaling pathway in dermal wound healing. Plos One. 2013;8:e64434-e.

40. Li J, Dong J, Zhang ZH, Zhang DC, You XY, Zhong Y, et al. miR-10a restores human mesenchymal stem cell differentiation by repressing KLF4. J Cell Physiol. 2013;228:2324-36.

41. Li RY, Chen LC, Zhang HY, Du WZ, Feng Y, Wang HB, et al. MiR-139 inhibits Mcl-1 expression and potentiates TMZ-induced apoptosis in glioma. CNS Neurosci Ther. 2013;19:477-83.

42. Moon JM, Xu L, Giffard RG. Inhibition of microRNA-181 reduces forebrain ischemia-induced neuronal loss. J Cereb Blood Flow Metab. 2013;33:1976-82.

43. He Q, Zhou X, Li S, Jin Y, Chen Z, Chen D, et al. MicroRNA-181a suppresses salivary adenoid cystic carcinoma metastasis by targeting MAPK-Snai2 pathway. Biochim Biophys Acta. 2013;1830:5258-66.

44. $X u Y Y, W u H J, M a ~ H D, X u L P$, Huo Y, Yin LR. MicroRNA-503 suppresses proliferation and cell-cycle progression of endometrioid endometrial cancer by negatively regulating cyclin D1. FEBS J. 2013;280:3768-79.

45. Wong TS, Liu XB, Wong YH, Ng WM, Yuen PW, Wei WI. Mature miR-184 as Potential Oncogenic microRNA of Squamous Cell Carcinoma of Tongue. Clin Cancer Res. 2008;14:2588-92.

46. Lynn FC. Meta-regulation: microRNA regulation of glucose and lipid metabolism. Trends Endocrinol Metab. 2009;20:452-9.

47. Tate R, Rotondo D, Davidson J. Regulation of lipid metabolism by microRNAs. Curr Opin Lipidol. 2015;26:243-4.

48. Fernandez-Hernando C, Suarez Y, Rayner KJ, Moore KJ. MicroRNAs in lipid metabolism. Curr Opin Lipidol. 2011;22:86-92.

49. Dávalos A, Goedeke L, Smibert P, Ramírez CM, Warrier NP, Andreo U, et al. miR-33a/b contribute to the regulation of fatty acid metabolism and insulin signaling. Proc Natl Acad Sci U S A. 2011;108:9232-7.

50. Esau C, Davis S, Murray SF, Xing XY, Pandey SK, Pear M, et al. miR-122 regulation of lipid metabolism revealed by in vivo antisense targeting. Cell Metab. 2006;3:87-98.

51. Nakanishi N, Nakagawa Y, Tokushige N. The up-regulation of microRNA-335 is associated with lipid metabolism in liver and white adipose tissue of genetically obese mice. Biochem Biophys Res Commun. 2009;385:492-6.

52. Horie T, Ono KH. MicroRNA-133 regulates the expression of GLUT4 by targeting KLF15 and is involved in metabolic control in cardiac myocytes. Biochem Biophys Res Commun. 2009;389:315-20.

53. Liang J, Liu C, Qiao A, Cui Y, Zhang H, Cui A, et al. MicroRNA-29a-c decrease fasting blood glucose levels by negatively regulating hepatic gluconeogenesis. J Hepatol. 2013;58:535-42.

54. Fei X, Qi M, Wu B, Song Y, Wang Y, Li T. MicroRNA-195-5p suppresses glucose uptake and proliferation of human bladder cancer T24 cells by regulating GLUT3 expression. Febs Letters. 2012;586:392-7.

55. Lin XZ, Luo J, Zhang LP, Wang W, Shi HB, Zhu JJ. miR-27a suppresses triglyceride accumulation and affects gene mRNA expression associated with fat metabolism in dairy goat mammary gland epithelial cells. Gene. 2013;521:15-23.

\section{Submit your next manuscript to BioMed Central and take full advantage of:}

- Convenient online submission

- Thorough peer review

- No space constraints or color figure charges

- Immediate publication on acceptance

- Inclusion in PubMed, CAS, Scopus and Google Scholar

- Research which is freely available for redistribution

Submit your manuscript at www.biomedcentral.com/submit 